\title{
Surgical treatment of paravalvular leak: Long-term results in a single-center experience (up to 14 years)
}

\author{
Maurizio Taramasso, MD, ${ }^{\mathrm{a}, \mathrm{b}}$ Francesco Maisano, MD, FESC, ${ }^{\mathrm{a}}$ Paolo Denti, MD, ${ }^{\mathrm{b}}$ Andrea Guidotti, Eng, ${ }^{\mathrm{a}}$ \\ Alessandro Sticchi, MD, ${ }^{\mathrm{b}}$ Alberto Pozzoli, MD, ${ }^{\mathrm{b}}$ Nicola Buzzatti, MD, ${ }^{\mathrm{b}}$ Michele De Bonis, MD, \\ Giovanni La Canna, MD, ${ }^{\mathrm{b}}$ and Ottavio Alfieri, MD, FETCS, FESC ${ }^{\mathrm{b}}$
}

Objectives: The aim of this study is to report the long-term outcomes (median follow-up time, 7 years; range, 1 month to 14 years) of patients who underwent surgery for paravalvular leak in our single-center experience.

\begin{abstract}
Methods: From October 2000 to November 2007, 122 consecutive patients underwent surgery for symptomatic paravalvular leak (40 patients with aortic paravalvular leak; 82 with mitral paravalvular leak). In 7 patients $(5.7 \%$, all mitral), surgery was performed on the beating heart through a right thoracotomy. In $35 \%$ of patients, multiple paravalvular leaks were present.
\end{abstract}

\begin{abstract}
Results: The mean age of patients was $62 \pm 11$ years, and European System for Cardiac Operative Risk Evaluation II was 7.2\% $\pm 6 \%$. Most of the patients were in New York Heart Association functional class III or IV $(60 \%)$. Symptomatic hemolysis was present in $31 \%$ of the patients, and $41 \%$ of the patients had more than 1 previous cardiac operation. Paravalvular leak repair was feasible in 79 patients $(65 \%)$, whereas in 43 patients (35\%) prosthesis re-replacement was required. Thirty-day mortality was $10.7 \%(13 / 122$ patients; $5 \%$ for aortic paravalvular leak and $13 \%$ for mitral paravalvular leak; $P=.1)$; 2 patients $(1.6 \%)$ with residual severe mitral paravalvular leak underwent successful redo surgery before discharge. Median length of stay was 7 days. Overall actuarial survival was $39 \% \pm 6 \%$ at 12 years; freedom from cardiac death was $54 \% \pm 7 \%$ at 12 years. Only 1 patient underwent redo surgery during follow-up. Multivariable analysis identified preoperative chronic renal failure (hazard ratio, $2.6 ; 95 \%$ confidence interval, $1.4-4.9 ; P=.03$ ) and more than 1 previous cardiac reoperation (hazard ratio, $2.3 ; 95 \%$ confidence interval, $1.3-4 ; P=.03$ ) as independent predictors of death at follow-up.

Conclusions: The operative mortality of surgical treatment of paravalvular leak is still high. Long-term outcomes remain suboptimal in these challenging patients, especially in the presence of multiple previous cardiac operations and associated co-pathologies. These results support the importance of alternative therapeutic options. (J Thorac Cardiovasc Surg 2015;149:1270-5)
\end{abstract}

See related commentary pages $1276-7$.

Paravalvular leak (PVL) is a common complication after surgical valve replacement, with reported incidences at follow-up of $2 \%$ to $17 \%$ in both mitral and aortic positions. ${ }^{1-3}$ Among patients in whom PVL develops after surgery, approximately $3 \%$ require reoperations because of heart failure, hemolysis, or a combination of both. ${ }^{4-6}$ In symptomatic patients, surgical treatment is associated with improved event-free survival compared with conservative management. ${ }^{3}$ The standard therapy for these defects is

From the Klinik für Herz- und Gefässchirurgie, Cardiac Surgery Department, ${ }^{\mathrm{a}}$ UniversitätsSpital Zürich, Zürich, Switzerland; and Cardiac Surgery Department, ${ }^{\mathrm{b}}$ San Raffaele University Hospital, Milan, Italy.

Disclosures: Authors have nothing to disclose with regard to commercial support.

Received for publication Oct 18, 2014; revisions received Nov 28, 2014; accepted for publication Dec 15, 2014; available ahead of print Jan 31, 2015.

Address for reprints: Maurizio Taramasso, MD, Klinik für Herz- und Gefässchirurgie, UniversitätsSpital Zürich, Rämistrasse, 100, 8091 Zürich, Switzerland (E-mail: Maurizio.Taramasso@usz.ch).

$0022-5223 / \$ 36.00$

Copyright (C) 2015 by The American Association for Thoracic Surgery

http://dx.doi.org/10.1016/j.jtcvs.2014.12.041 surgical closure of PVL or valve re-replacement. However, redo surgery in this context is often associated with high morbidity and mortality rates, as well as a high risk of leak recurrence. ${ }^{1,7}$ Limited data exist on the long-term outcomes of surgical treatment of PVLs.

We recently reported the acute results of surgical treatment of PVLs compared with transapical transcatheter closure. ${ }^{8}$ The aim of this study is to report the long-term results (up to 14 years) of the surgical treatment of PVLs in our single-center experience.

\section{MATERIALS AND METHODS}

We retrospectively analyzed the clinical and echocardiographic data of a cohort of consecutive patients who underwent surgery for symptomatic PVL, without associated procedures, between October 2000 and November 2007. Clinical, echocardiographic, operative, and outcome data were collected prospectively. All patients underwent preoperative coronary angiography and transesophageal Doppler echocardiography. Periprosthetic PVL was defined as a regurgitant jet, demonstrated by transesophageal echocardiography, originating between the outer margin of the prosthetic sewing ring and the native tissues around the valve. The severity of the PVL was defined as mild $(1+/ 4+)$, moderate $(2+/ 4+)$, moderate to severe $(3+/ 4+)$, and severe $(4+/ 4+)$ using an integrative approach, as recommended by the current guidelines. ${ }^{9}$ All patients included in the study had preoperative moderate-to-severe or severe PVL. 


\section{Abbreviations and Acronyms \\ NYHA $=$ New York Heart Association \\ PVL = paravalvular leak}

Patients were considered for surgery if 1 or more of the following criteria were met: severe symptoms of heart failure (New York Heart Association [NYHA] class III-IV or class II with significant lifestyle impairment), clinically significant hemolytic anemia, and moderately severe or severe paravalvular prosthetic regurgitation. Symptomatic hemolysis was defined as hemolytic anemia (hemoglobin $\leq 10 \mathrm{~g} / \mathrm{dL}$, lactate dehydrogenase $\geq 600 \mathrm{mg} / \mathrm{dL}$, haptoglobin $\leq 10 \mathrm{mg} / \mathrm{dL}$ ) requiring more than 2 units of blood transfusions or erythropoietin injections within 90 days to maintain hemoglobin $10 \mathrm{~g} / \mathrm{dL}$ or greater, without any other source of blood loss. ${ }^{10}$ Patients with associated prosthetic dysfunction and patients with active acute endocarditis were excluded from this study. Figure 1 shows the patient flow and final study population.

The study protocol was performed in accordance with the institutional ethics committee, and all patients gave informed written consent for the procedures. The need for consent to participate in this research study was waived in view of its observational, retrospective, and anonymous nature.

\section{Patient Characteristics}

A total of 122 patients were treated; 82 patients had mitral PLV (67.2\%) and 40 patients $(32.8 \%)$ had aortic PVL. In 35 patients (28.7\%), multiple PVLs were present.

The mean age of the overall study population was $62 \pm 11$ years. Predicted surgical risk with European System for Cardiac Operative Risk Evaluation II was 7.2\% $\pm 6 \%$. Most of the patients were in NYHA functional class III or IV (overall $60 \%$; $66 \%$ with mitral PVL and $45 \%$ with aortic PVL; $P=.02$ ). Patients with mitral PVL have a higher prevalence of atrial fibrillation $(P=.001)$. Symptomatic hemolysis was present in 37 patients $(30.3 \%)$ and was particularly frequent in patients with mitral PVL ( $41 \%$ vs $13 \% ; P=.0009)$. Many patients had more than 1 previous cardiac operation ( $40.9 \%$ overall; $50 \%$ for mitral PVL and $22 \%$ for aortic PVL; $P=.003$ ). Demographic and clinical features of the patients are detailed in Table 1.

\section{Surgical Treatment}

The majority of the patients underwent surgery through a midline sternotomy and moderate hypothermic cardiopulmonary bypass. A minority of the patients with isolated mitral PVL underwent mitral surgery on the beating heart via a right thoracotomy (7 patients). In case of mitral PVL, the mitral prosthesis was approached through a conventional left atriotomy in all patients; in case of aortic PVL, the aortic prosthesis was approached through a transversal aortotomy. After the inspection of the prosthesis and the identification of the PVL, repair was performed with interrupted Ticron 2-0 sutures with pledgets. If repair was judged unfeasible by the surgeon, replacement of the prosthesis was performed. The use of interrupted over running suture for valve replacement depended on the surgeon's preference.

\section{Statistical Analysis}

Statistical analysis was conducted using SPSS 22.0 software (IBM SPSS Statistics for Macintosh, Version 22.0. IBM Corp, Armonk, NY). Continuous variables are presented as mean \pm standard deviation, and categoric variables are expressed as percentages. Univariable comparisons have been performed with the Student unpaired $t$ test for continuous normally distributed data, which have been tested by the Shapiro-Wilk normality test; the Mann-Whitney rank-sum test was used for comparisons of non-normally distributed data, and the chi-square test was used for categoric data. Long-term survival was presented using the Kaplan-Meier

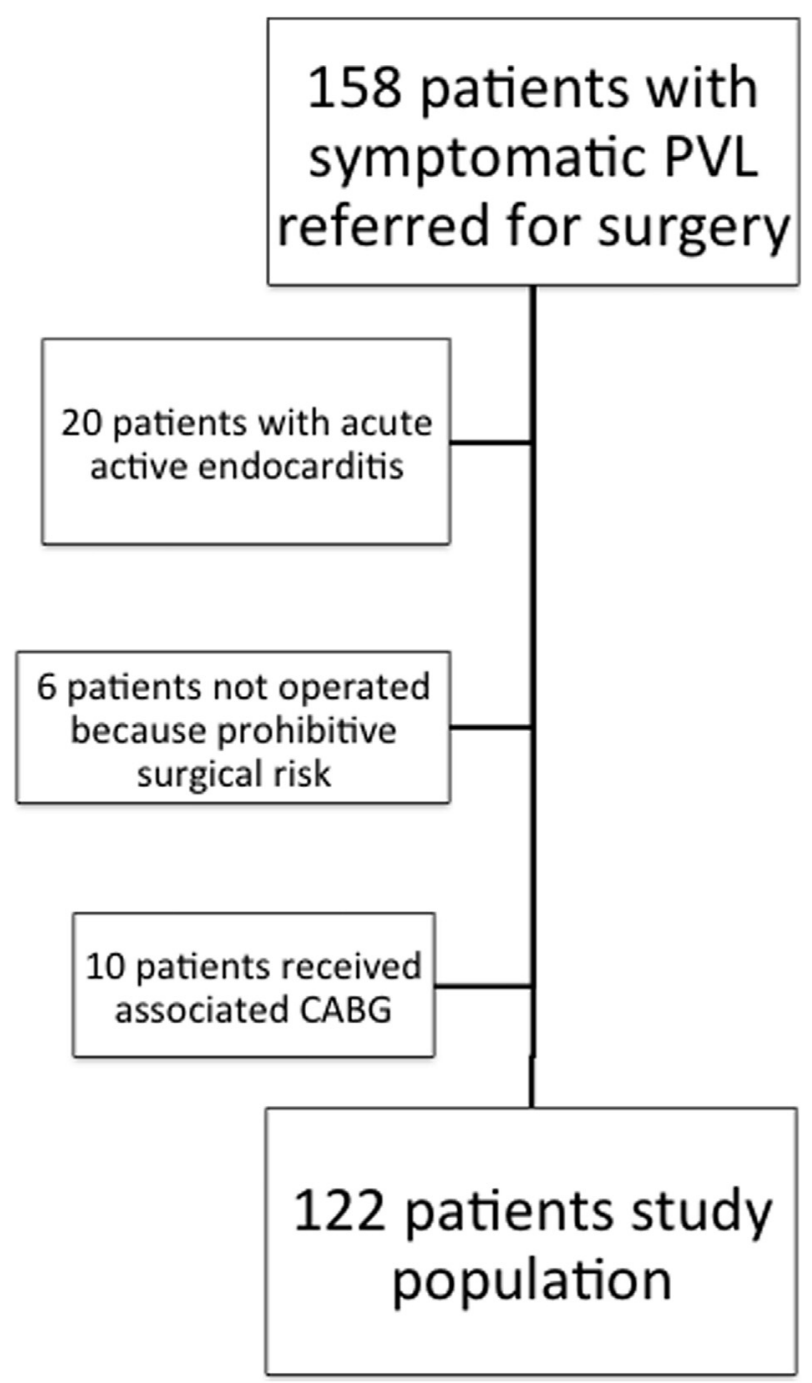

FIGURE 1. Diagram showing patient flow and final study populations. $C A B G$, Coronary artery bypass grafting; $P V L$, paravalvular leak.

method; comparisons were performed with the log-rank test. Multivariable Cox proportional-hazards regression modeling was performed to determine the independent predictors of mortality at follow-up using purposeful selection of covariates. Variables associated at univariate analysis with death at follow-up (all with a $P<.1$ ) and those judged to be of clinical importance from previous published literature were eligible for inclusion in the multivariable model-building process.

\section{RESULTS}

\section{Procedural and In-Hospital Outcomes}

Acute procedural success was $98 \%$ (120/122 patients; 2 patients had residual severe mitral PVL and required redosurgery before discharge). PVL repair was feasible in 79 patients $(65 \%)$, and prosthesis re-replacement was required in 43 patients $(35 \%)$.

Thirty-day mortality was $10.7 \%$ (13/122 patients; 7 patients died of acute cardiogenic shock, 4 patients died of multiorgan failure secondary to sepsis, 1 patient died of 
TABLE 1. Preoperative clinical features of the study population

\begin{tabular}{|c|c|c|c|c|}
\hline & Overall $(n=122)$ & Mitral PVL $(n=82)$ & Aortic PVL $(n=40)$ & $P$ value* \\
\hline Age (y) & $61.9 \pm 11$ & $62 \pm 11$ & $60 \pm 13$ & .3 \\
\hline Female gender (n, \%) & $40(33)$ & $32(39)$ & $8(20)$ & .03 \\
\hline euroSCORE II ( $\%)$ & $7.2 \pm 6$ & $7.5 \pm 8$ & $6.9 \pm 5$ & .4 \\
\hline No. of previous cardiac surgeries (mean $\pm \mathrm{SD}$ ) & $1.7 \pm 1$ & $1.9 \pm 1$ & $1.2 \pm 0.7$ & .001 \\
\hline$>1$ reoperation $(\mathrm{n}, \%)$ & $50(41)$ & $41(50)$ & $9(22)$ & .003 \\
\hline Peripheral artery disease (n, \%) & $11(9)$ & $5(6)$ & $6(15)$ & .1 \\
\hline Previous endocarditis (n, \%) & $33(27)$ & $18(22)$ & $15(37)$ & .06 \\
\hline Atrial fibrillation (n, \%) & $73(59)$ & $57(70)$ & $16(40)$ & .001 \\
\hline Chronic renal failure (n, \%) & $18(15)$ & $11(13)$ & $7(17)$ & .6 \\
\hline $\operatorname{COPD}(\mathrm{n}, \%)$ & $14(11)$ & $11(14)$ & $3(7)$ & .3 \\
\hline Symptomatic hemolysis (n, \%) & $37(30)$ & $32(39)$ & $5(13)$ & .0009 \\
\hline Diabetes (n, \%) & $18(15)$ & $13(16)$ & $5(13)$ & .6 \\
\hline NYHA functional class III-IV (n, \%) & $70(57)$ & $52(63)$ & $18(45)$ & .02 \\
\hline $\mathrm{sPAP}>40 \mathrm{~mm} \mathrm{Hg}(\mathrm{n}, \%)$ & $60(49)$ & $43(52)$ & $17(42)$ & .2 \\
\hline $\operatorname{LVEF}($ mean $\pm \mathrm{SD})$ & $58.9 \pm 5$ & $57.1 \pm 8$ & $59.5 \pm 3$ & .3 \\
\hline
\end{tabular}

Chronic renal failure was defined as a baseline glomerular filtration rate $\leq 60 \mathrm{~mL}$. COPD, Chronic obstructive pulmonary disease; euroSCORE, European System for Cardiac Operative Risk Evaluation; $L V E F$, left ventricular ejection fraction; NYHA, New York Heart Association; $P V L$, paravalvular leak; $S D$, standard deviation; $s P A P$, systolic pulmonary artery pressure. *Student unpaired $t$ test for continuous data; chi-square test for categoric data.

perioperative acute myocardial infarction, 1 patient died of bleeding complications). In particular, 30-day mortality was $8 \%$ for patients at the first re-sternotomy, $12 \%$ at the second re-sternotomy, $16 \%$ at the third re-sternotomy, and $25 \%$ at the fourth re-sternotomy. No 30-day deaths occurred among the 3 patients at the fifth re-sternotomy. Among the patients who died within 30 days, 11 had mitral PVL and 2 had aortic PVL $(P=.1)$. Postoperative median mean length of stay was 7 days (interquartile range, 5-13), with no differences between mitral and aortic PVLs.

Postprocedural echocardiography showed a residual significant PVL in 2 patients with mitral PVL who received initial PVL repair and required redo surgery within the same admission. All of the other patients had less than mild residual PVL. Table 2 summarizes the periprocedural results. Table 3 compares the characteristics of 30-day survivors with 30-day deaths. No significant differences were observed.

\section{Long-Term Results}

Median follow-up was 62 months (range, 1 month to 14 years). Overall, actuarial survival at follow-up was 39.3\% $\pm 6 \%$ at 12 years (Figure 2). Long-term overall actuarial survival was significantly reduced in patients who had more than 1 cardiac reoperation $(39.4 \% \pm 6 \%$ vs $64 \% \pm$ $6 \%$ at 9 years; $P=.002$ ) (Figure 3 ) and in patients with preoperative chronic renal failure $(54 \% \pm 11 \%$ vs $67 \% \pm 5 \%$ at 6.5 years; $P=.001$ ) (Figure 4 ). A tendency toward a reduced survival was observed in patients with mitral PVL compared with those with aortic PVL $(49 \% \pm 6 \%$ vs $63 \% \pm 9 \%$ at 8.5 years; $P=.07$ ). Only 1 patient underwent redo surgery during follow-up. Overall freedom from cardiac death was $54 \% \pm 7 \%$ at 12 years and was significantly reduced in patients with mitral PVL $(56 \% \pm 6 \%$ for mitral PVL compared with $78 \% \pm 9 \%$ for aortic PVL at 12 years; $P=.01$ ) (Figure 5). No differences in terms of cardiac death were observed at follow-up according to the type of suture techniques $(P=.3)$ or repair versus rereplacement $(P=.5)$.

Univariate and multivariate analyses of risk factors for mortality at follow-up are shown in Table 4. Candidate variables in the multivariate model included chronic renal failure, more than 1 previous cardiac operation, and mitral PVL. Preoperative chronic renal failure (defined as a baseline glomerular filtration rate $\leq 60 \mathrm{~mL}$ ) (hazard ratio, 2.6; $95 \%$ confidence interval, $1.4-4.9 ; P=.03$ ) and more than

TABLE 2. Perioperative results

\begin{tabular}{|c|c|c|c|c|}
\hline & Overall $(n=122)$ & Mitral PVL $(\mathbf{n}=\mathbf{8 2})$ & Aortic PVL $(n=40)$ & $P$ value* \\
\hline 30-d mortality (n, \%) & $13(10.7)$ & $11(13)$ & $2(5)$ & .1 \\
\hline Acute procedural success $(\mathrm{n}, \%)$ & $120(98)$ & $80(97)$ & $40(100)$ & .4 \\
\hline Severe acute kidney injury (n, \%) & $5(4)$ & $4(5)$ & $1(2.5)$ & .2 \\
\hline $\operatorname{LCOS}(n, \%)$ & $6(5)$ & $5(6)$ & $1(2.5)$ & .1 \\
\hline Septic shock (n, \%) & $6(5)$ & $5(6)$ & $1(2.5)$ & .1 \\
\hline Major stroke $(\mathrm{n}, \%)$ & $1(0.7)$ & 0 & $1(2.5)$ & .3 \\
\hline $\operatorname{AMI}(\mathrm{n}, \%)$ & $1(0.7)$ & $1(1)$ & 0 & .4 \\
\hline Residual significant PVL at discharge (n, \%) & $2(1)$ & $2(2.4)$ & 0 & .2 \\
\hline
\end{tabular}

$A M I$, Acute myocardial infarction; $L C O S$, low cardiac output syndrome; $P V L$, paravalvular leak. *Chi-square test. 
TABLE 3. Univariate analysis of 30-day survivors compared with 30day death

\begin{tabular}{lccc}
\hline & $\begin{array}{c}\text { 30-d survivors } \\
(\mathbf{n}=\mathbf{1 0 9})\end{array}$ & $\begin{array}{c}\text { 30-d deaths } \\
(\mathbf{n}=\mathbf{1 3})\end{array}$ & $\boldsymbol{P}$ value* \\
\hline Age (y) & $61.6 \pm 12$ & $64.4 \pm 12$ & .4 \\
Female gender (n, \%) & $37(34)$ & $3(23)$ & .4 \\
euroSCORE II (\%) & $7.5 \pm 3$ & $7.1 \pm 4$ & .6 \\
Mitral PVL (n, \%) & $71(65)$ & $11(84)$ & .1 \\
$>$ 1 reoperation (n, \%) & $43(39)$ & $7(53)$ & .3 \\
Peripheral artery disease (n, \%) & $11(10)$ & 0 & .1 \\
Previous endocarditis (n, \%) & $28(26)$ & $5(38)$ & .3 \\
Atrial fibrillation (n, \%) & $67(61)$ & $6(46)$ & .3 \\
Chronic renal failure (n, \%) & $14(13)$ & $4(31)$ & .06 \\
COPD (n, \%) & $13(12)$ & $1(8)$ & .7 \\
Symptomatic hemolysis (n, \%) & $34(31)$ & $3(23)$ & .8 \\
Diabetes (n, \%) & $16(15)$ & $2(16)$ & .9 \\
NYHA functional class III-IV & $64(58)$ & $6(46)$ & .7 \\
$\quad(n, \%)$ & & & \\
sPAP $>40$ mm Hg (n, \%) & $55(50)$ & $5(38)$ & .5 \\
LVEF (mean \pm SD) & $58 \pm 9$ & $57 \pm 4$ & .8 \\
\hline
\end{tabular}

Chronic renal failure was defined as a baseline glomerular filtration rate $\leq 60 \mathrm{~mL}$. COPD, Chronic obstructive pulmonary disease; euroSCORE, European System for Cardiac Operative Risk Evaluation; $L V E F$, left ventricular ejection fraction; $N Y H A$, New York Heart Association; $P V L$, paravalvular leak; $S D$, standard deviation; $s P A P$, systolic pulmonary artery pressure. *Student unpaired $t$ test for continuous data; chi-square test for categoric data.

1 previous cardiac reoperation (hazard ratio, $2.3 ; 95 \%$ confidence interval, $1.3-4 ; P=.03$ ) were identified as independent predictors of mortality at follow-up.

\section{DISCUSSION}

Surgical reoperation is the standard treatment for symptomatic PVLs. ${ }^{3,5,6}$ It has been demonstrated that in symptomatic patients with PVL, surgical treatment is associated with improved survival compared with conservative management. ${ }^{3}$ However, redo surgery is often associated with high morbidity and mortality: several series

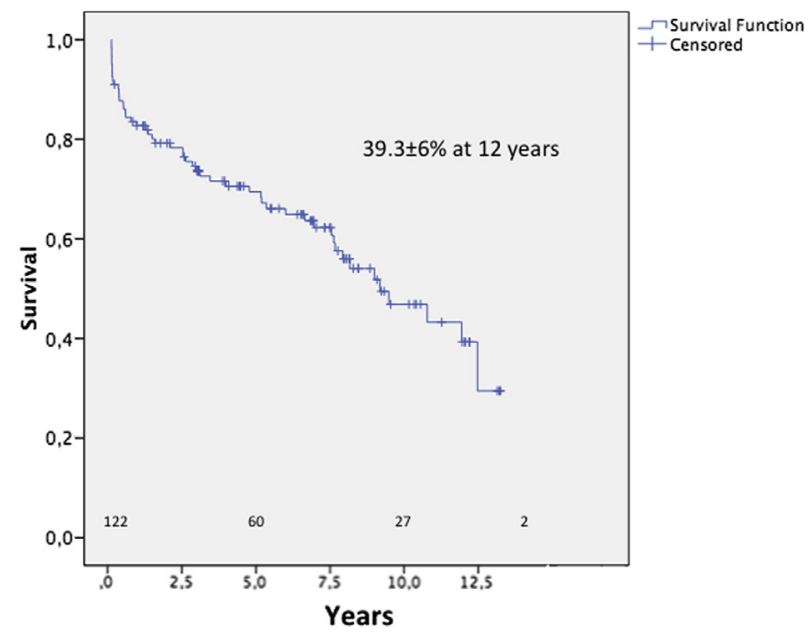

FIGURE 2. Overall actuarial survival at follow-up.

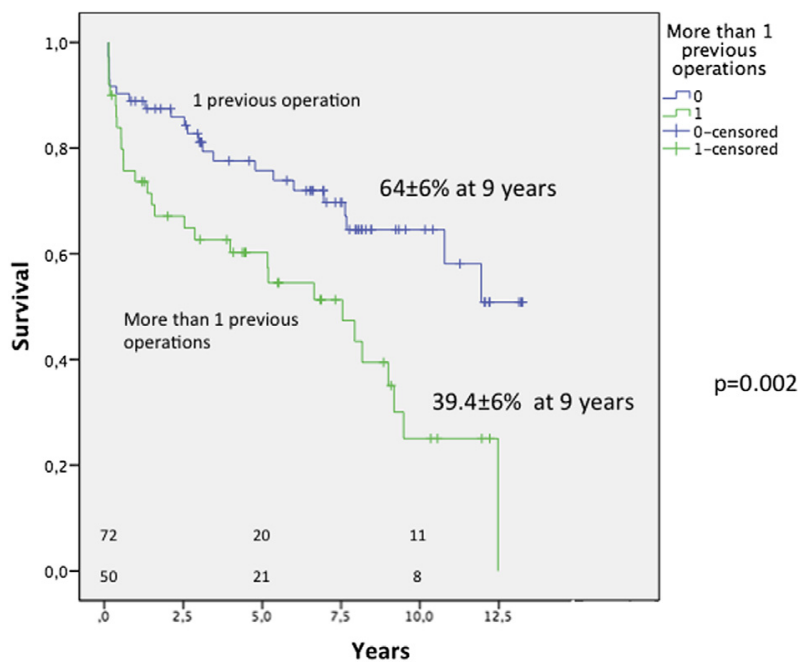

FIGURE 3. Survival at follow-up according to the number of previous open operations.

report an acute mortality between $6 \%$ and $22 \%$ after surgical reoperation for PVL. ${ }^{3,5-7}$ Increased risk was observed in severely symptomatic patients (NYHA class III-IV and severe hemolysis) and in patients with multiple surgical reinterventions. $^{3,5}$ Associated co-pathologies could further increase operative risk. To date, long-term longitudinal outcomes after surgical treatment of PVL are largely unknown. We report the long-term results (up to 14 years) of the surgical treatment of PVL of a large series of patients from a single high-volume center experience. Patients with mitral PVL were in slightly worse baseline clinical condition and were significantly more symptomatic compared with patients with aortic PVL. The present study confirms that surgery is an effective option for the treatment of PVL. Procedural success was $98 \%$, and in patients with wide and multiple leaks it is possible to achieve optimal anatomic results. Redo surgery for recurrent PVL was required during follow-up in only 1 patient. However, in the absence of a rigorous echocardiographic follow-up, freedom from reoperation may underestimate the rate of PVL recurrence, because many patients with recurrence could not have undergone reoperation because of the prohibitive risk of redo surgery.

In regard to safety, the 30-day mortality reported in the present study is relatively high $(>10 \%$, all cardiac-related deaths), confirming that surgery in the context of patients with PVL is still a high-risk procedure. Patients with PVL are usually patients with multiple previous open operations, with associated co-pathologies and severely symptomatic. In view of the high-risk profile of the patients included in the present series, a $10 \%$ of acute mortality may be considered acceptable, being comparable to that reported in other surgical series. Of note, we did not identify any specific subgroup of patients with significantly higher risk for 30-day 


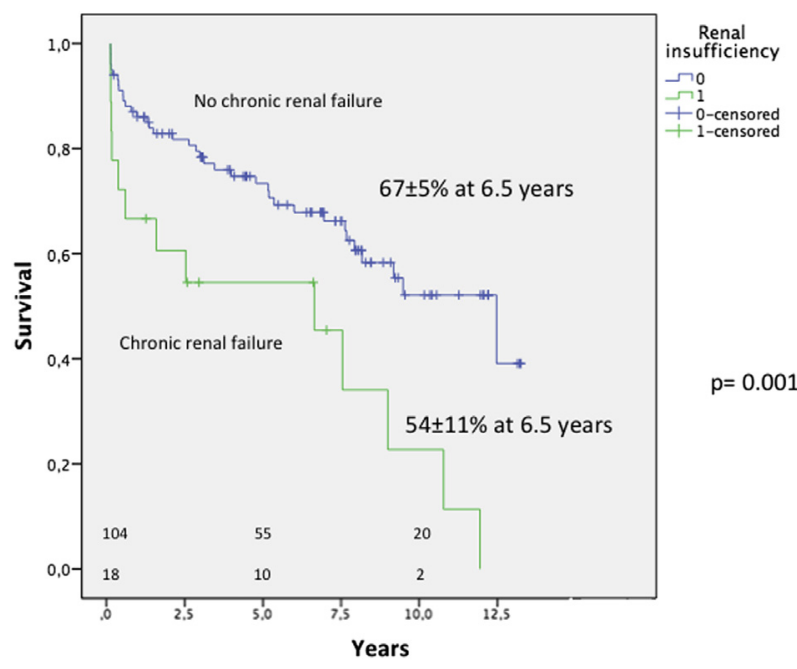

FIGURE 4. Survival at follow-up according to baseline chronic renal failure (defined as glomerular filtration rate $\leq 60 \mathrm{~mL}$ ).

mortality. A trend toward a higher mortality in mitral patients was observed, reflecting the more compromised clinical conditions of the patients with mitral PVL.

The most important finding of this study is that long-term results of conventional surgery in this context are largely suboptimal. Overall actuarial survival at 12 years was less than $40 \%$, and this is particularly significant considering the relatively young age of the patients included in the study ( $<62$ years on average). This aspect may be partially explained by the high-risk preoperative profile. In particular, preoperative chronic renal failure and the presence of more than 1 previous cardiac operation were independently associated with increased risk of mortality at follow-up. Patients with mitral PVL had a higher cardiac-related mortality at follow-up compared with patients with aortic PVL.

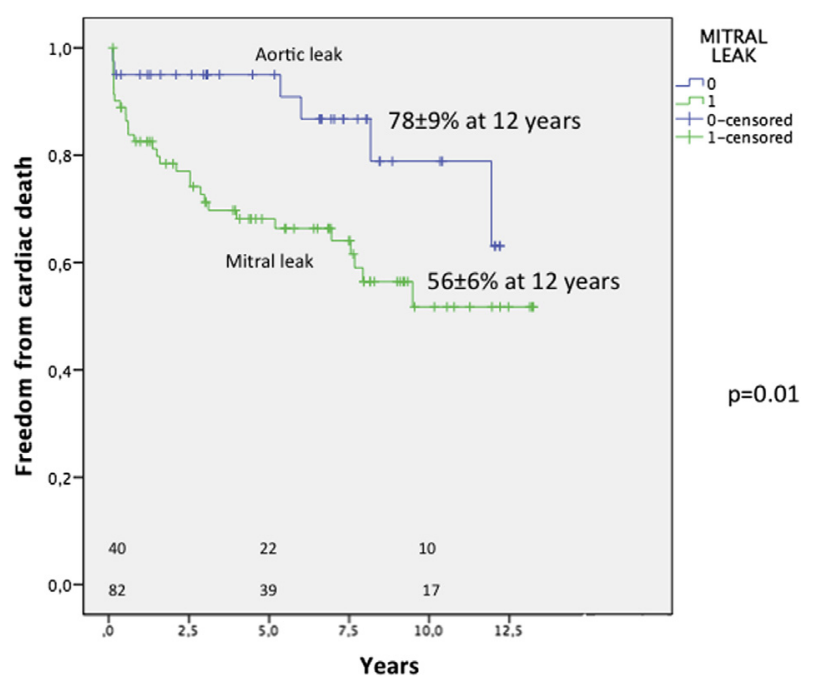

FIGURE 5. Freedom from cardiac death in aortic and mitral PVLs.
TABLE 4. Univariate and multivariate Cox regression analyses of risk factors for mortality at follow-up

\begin{tabular}{|c|c|c|c|c|}
\hline & \multicolumn{2}{|c|}{ Univariate } & \multicolumn{2}{|c|}{ Multivariate } \\
\hline & HR $(95 \%$ CI $)$ & $P$ value & HR $(95 \%$ CI $)$ & $P$ value \\
\hline Chronic renal failure & $2.7(1.4-5.1)$ & .02 & $2.6(1.4-4.9)$ & .03 \\
\hline$>1$ redo & $2.26(1.3-2.9)$ & .03 & $2.3(1.3-4)$ & .03 \\
\hline Mitral PVL & $1.7(0.9-3.4)$ & .07 & $1.5(0.7-3)$ & .2 \\
\hline Previous endocarditis & $1.4(0.7-2.7)$ & .3 & & \\
\hline COPD & $1.6(0.8-3.3)$ & .2 & & \\
\hline NYHA class III-IV & $1.7(0.9-3)$ & .2 & & \\
\hline Symptomatic hemolysis & $0.8(0.4-1.6)$ & 6 & & \\
\hline $\mathrm{sPAP}>40 \mathrm{~mm} \mathrm{Hg}$ & $1(0.6-1.7)$ & .9 & & \\
\hline Atrial fibrillation & $1(0.6-1.8)$ & .8 & & \\
\hline
\end{tabular}

Bold indicates significant values. Chronic renal failure was defined as a baseline glomerular filtration rate $\leq 60 \mathrm{~mL}$. CI, Confidence interval; COPD, chronic obstructive pulmonary disease; $H R$, hazard ratio; NYHA, New York Heart Association; $P V L$, paravalvular leak; $S P A P$, systolic pulmonary artery pressure.

Although preoperative left ventricular ejection fraction was preserved in both aortic and mitral PVL cases, patients with mitral valve disease more frequently have a certain degree of associated left ventricular dysfunction that could affect long-term outcomes. Moreover, the natural history of patients with a prosthetic valve in the mitral position is usually worse if compared with the natural course of patients with an aortic prosthesis. ${ }^{1}$ Accordingly, we observed a significantly higher cardiac-related mortality in patients with mitral PVL.

The high 30-day mortality and the disappointing longterm results that we observed strongly point out the need for a valid therapeutic option alternative to conventional surgery, mainly in the patients presenting with risk factors for increased mortality (patients with multiple previous cardiac operation, associated chronic renal failure, and mitral PVL). Percutaneous PVL closure has been proposed as an attractive less-invasive option and was found to alleviate the consequences and symptoms of PVLs in high-risk patients. ${ }^{11}$

Transcatheter closure of mitral PVL is more challenging than aortic PVL and is mostly performed through a transseptal approach. However, percutaneous mitral PVL closure is challenging from a technical point of view and requires a very experienced and skilled operator. Reproducibility remains a major concern: Technical success rates range from $60 \%$ to $90 \%$ in different series. ${ }^{10,12-17}$ Transapical access represents an attractive alternative solution for transcatheter mitral PVL closure, ensuring by definition coaxiality to the mitral plane and direct engagement to the leak. ${ }^{18}$ We recently reported satisfactory acute results of a small series of 17 patients who underwent mitral PVL closure through the transapical route. The 30day mortality was $0 \%$, with an acute procedural success of $94 \%{ }^{8}$

Although the safety and efficacy of transapical PVL repair have never been compared with surgical reoperation 
